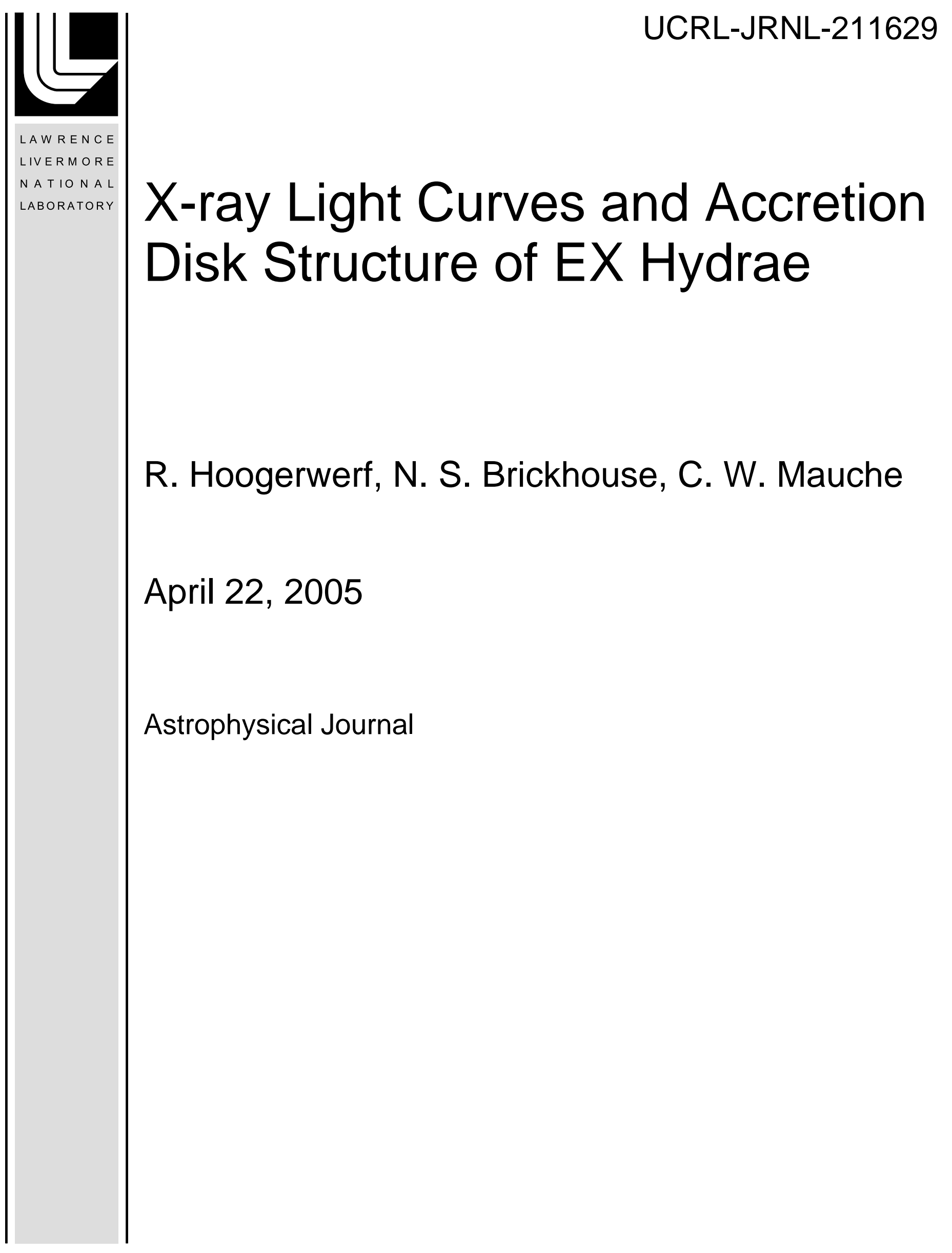


This document was prepared as an account of work sponsored by an agency of the United States Government. Neither the United States Government nor the University of California nor any of their employees, makes any warranty, express or implied, or assumes any legal liability or responsibility for the accuracy, completeness, or usefulness of any information, apparatus, product, or process disclosed, or represents that its use would not infringe privately owned rights. Reference herein to any specific commercial product, process, or service by trade name, trademark, manufacturer, or otherwise, does not necessarily constitute or imply its endorsement, recommendation, or favoring by the United States Government or the University of California. The views and opinions of authors expressed herein do not necessarily state or reflect those of the United States Government or the University of California, and shall not be used for advertising or product endorsement purposes. 


\title{
X-RAY LIGHT CURVES AND ACCRETION DISK STRUCTURE OF EX HYDRAE
}

\author{
R. Hoogerwerf, N. S. Brickhouse \\ Smithsonian Astrophysical Observatory, Harvard-Smithsonian Center for Astrophysics, 60 \\ Garden Street, MS 31, Cambridge, MA 02138 \\ rhoogerwerf@cfa.harvard.edu, nbrickhouse@cfa.harvard.edu \\ and \\ C. W. Mauche \\ Lawrence Livermore National Laboratory, L-473, 7000 East Avenue, Livermore, CA 94550 \\ mauche@cygnus. Ilnl.gov
}

\begin{abstract}
We present X-ray light curves for the cataclysmic variable EX Hydrae obtained with the Chandra High Energy Transmission Grating Spectrometer and the Extreme Ultraviolet Explorer Deep Survey photometer. We confirm earlier results on the shape and amplitude of the binary light curve and discuss a new feature: the phase of the minimum in the binary light curve, associated with absorption by the bulge on the accretion disk, increases with wavelength. We discuss several scenarios that could account for this trend and conclude that, most likely, the ionization state of the bulge gas is not constant, but rather decreases with binary phase. We also conclude that photoionization of the bulge by radiation originating from the white dwarf is not the main source of ionization, but that it is heated by shocks originating from the interaction between the inflowing material from the companion and the accretion disk. The findings in this paper provide a strong test for accretion disk models in close binary systems.
\end{abstract}

Subject headings: novae, cataclysmic variables - stars: individual (EX Hydrae)— X-ray: stars 


\section{Introduction}

Cataclysmic variables (CVs) are semi-detached, interacting binaries consisting of a white dwarf and a late-type star (hereafter, the companion) that overflows its Roche Lobe. In most cases the overflowing material settles into a disk around the white dwarf before accreting onto its surface. At the point where the stream of material from the companion collides/interacts with the accretion disk a "hot-spot" or "bulge" forms. Much of our understanding of the three-dimensional structure of CV disks has been obtained from studying their light curves; for CVs seen nearly edge-on, light curves show broad modulations (either in emission or absorption, depending on the wavelength regime) and deep eclipses. The broad modulation is due to the hot-spot orbiting with the binary system and the eclipses are due to occultation of the white dwarf and/or hot-spot by the companion (see e.g., Wood et al. 1986).

A subset of the CVs, the so-called magnetic CVs, contain white dwarfs with magnetic fields that are sufficiently strong to force the accreting material away from the orbital plane of the binary and onto one or both of the white dwarf's magnetic poles. The accreting material approaches the white dwarf supersonically and so passes through a stand-off shock before cooling and settling onto the surface of the white dwarf (Aizu 1973; Kylafis \& Lamb 1982). The material in the shock is hot $(\sim 10-100 \mathrm{keV})$, so magnetic CVs are powerful X-ray sources. Magnetic CVs are divided into two subclasses: polars and intermediate polars (IPs). In polars the stars are tidally locked; this "static" configuration results in an accretion flow from the inner Lagrangian point of the binary along the magnetic field lines of the white dwarf onto its magnetic pole(s). In IPs, where the white dwarf is spinning faster than the orbital period, matter also accretes onto the white dwarf along the magnetic field lines, but it first accumulates in an accretion disk surrounding the white dwarf.

In this paper we discuss the X-ray light curves of the IP EX Hydrae (hereafter, EX Hya) obtained with the Chandra X-ray Observatory (Chandra) High Energy Transmission Grating (HETG) spectrometer and the Extreme Ultraviolet Explorer (EUVE) Deep Survey (DS) photometer. EX Hya consists of a $0.49 \pm 0.13 \mathrm{M}_{\odot}$ white dwarf with a spin/rotation period of 67 minutes and a $0.078 \pm 0.014 \mathrm{M}_{\odot}$ companion (Fujimoto \& Ishida 1997; Beuermann et al. 2003; Hoogerwerf, Brickhouse, \& Mauche 2004). The binary system has an orbital period of 98 minutes and an inclination $i=77^{\circ}$. EX Hya light curves have been studied extensively in the optical (e.g., Vogt, Krzeminski, \& Sterken 1980; Sterken et al. 1983), UV (e.g., Mauche 1999; Belle et al. 2003), EUV (e.g., Hurwitz et al. 1997; Belle et al. 2002), and X-rays (e.g., Rosen, Mason, \& Córdova 1988; Allan, Hellier, \& Beardmore 1998).

The X-ray white dwarf light curve, i.e., the observed flux as a function of the white dwarf spin phase (hereafter $\phi_{67}$ ) shows a broad sinusoidal modulation that peaks around $\phi_{67} \approx 1.0$. Its amplitude increases with increasing wavelength of the emission. Two origins have been 
suggested for this broad modulation. One is the "accretion curtain" model (Rosen et al. 1988), in which the pre-shock material absorbs emission created in the post-shock region. Since the pre-shock material is confined to the magnetic field, its projected column density changes as the white dwarf rotates, hence the amount of absorption varies (see figures 7 and 8 of Rosen et al. 1988). The other is the occultation model (e.g., Allan et al. 1998; Mukai 1999), in which the $\phi_{67}$ modulation is due to occultation of the emission by the limb of the white dwarf.

The X-ray binary light curve of EX Hya, i.e., the observed flux as a function of binary phase (hereafter $\phi_{98}$ ), shows two main features: a broad modulation, thought to be due to photoelectric absorption of white dwarf and accretion column emission by the hot spot or bulge material on the accretion disk, and a sharp partial eclipse, due to the occultation of the lower accretion pole of the white dwarf by the companion (see e.g., Rosen et al. 1988; Mukai et al. 1998).

This paper focuses on the binary light curve using data obtained with Chandra and $E U V E$ and is organized as follows: $\S 2$ describes the data and the construction of the light curves, $\S 3$ presents the binary light curves and a model for the accretion disk bulge, $\S 4$ discusses the results, and $\S 5$ presents the conclusions.

\section{Observations and Reduction}

\section{Chandra}

EX Hya was observed by Chandra using the HETG in combination with the Advanced CCD Imaging Spectrometer in its spectroscopy layout (ACIS-S) on 2000 May 18 for $60 \mathrm{ks}$. The observation was continuous and covers $\sim 10$ orbital revolutions of the binary system and $\sim 15$ rotations of the white dwarf. We reduced the data using the Chandra Interactive Analysis of Observations (CIAO version 3.0) software package ${ }^{1}$, making only two departures from the standard reduction: (1) we turned randomization off to minimize any artificial line broadening (i.e., we set rand_pix_size equal to zero in the $t g_{-} r e s o l v e \_e v e n t s$ tool; note that in CIAO version 3.1 and later rand_pix_size $=0$ is the default setting), and (2) we applied a solar system barycentric correction so that the event times are in Barycentric Dynamical Time instead of spacecraft time.

Light curves were constructed using the CIAO tool dmextract. We note that since the

\footnotetext{
${ }^{1}$ http://cxc.harvard.edu/ciao/
} 
HETG grating spectra span several CCDs on the ACIS detector, it is recommended in the dmextract documentation ${ }^{2}$ that light curves be extracted for each CCD individually so that the correct Good Time Interval (GTI) table for each CCD is used (the GTIs usually differ from CCD to CCD). The individual CCD light curves can then be combined to form the correct light curve, for example, a spectral order light curve. For the EX Hya observation, the GTIs for ACIS CCDs \#4 through \#9 differed by less than $6.6 \mathrm{~s}$, or $\sim 0.01 \%$ of the $60 \mathrm{ks}$ integration time, so we chose to ignore this small difference and extract the light curves for spectral orders spanning several CCDs in the same extraction (in this case the first GTI table available to dmextract is used, i.e., the CCD \#7 GTI, which contains the zeroth order).

We generated light curves for the combined first orders of the High Energy Grating (HEG) and Medium Energy Grating (MEG) as a function of binary phase $\phi_{98}$ for the optical ephemeris of Hellier \& Sproats (1992). Furthermore, we created light curves for the following wavelength intervals: $1-5 \AA, 5-10 \AA, 10-15 \AA$, and $15-20 \AA$. In the remainder of this paper we refer to these light curves as $£(\lambda)$, where $\lambda$ indicates the wavelength range.

We also generated light curves for the HETG background and conclude that they are constant with time and account for less than $0.4 \%$ of the signal in the source light curves.

\section{EUVE}

EX Hya was observed by EUVE (Bowyer \& Malina 1992; Bowyer et al. 1994) between 2000 May 2 and 2000 June 15. The data were extracted from the HEASARC ${ }^{3}$ data archive in the form of 11 FITS-format events files, which were manipulated with custom IDL software developed over many years of EUVE observations of cataclysmic variables (see Mauche 2002, and references therein). Source counts were collected from a 2 arcmin radius circle centered on EX Hya, while background counts were collected from a surrounding annulus having an inner radius of 3 arcmin and an outer radius of 5.385 arcmin (such that the ratio of source to background areas was 1:5). After discarding numerous short $(\Delta t<100 \mathrm{~s})$ data intervals comprising less than $1 \%$ of the total exposure, we "manually" adjusted the start and stop times of the nominal good time intervals to include only those times when the source was above Earth's limb and the background and primbsch/deadtime correction was low. This filtering resulted in 719 good time intervals for a total exposure of $967 \mathrm{ks}$. Event times were corrected from spacecraft time to HJD, HJD was converted to binary phase using the ephemeris of Hellier \& Sproats (1992), phase-folded source $S$, background $B$, and primbsch-

\footnotetext{
${ }^{2}$ http://cxc.harvard.edu/ciao/why/lightcurve.html

${ }^{3}$ http://heasarc.gsfc.nasa.gov
} 
weighted exposure $\Delta t$ light curves were accumulated, and the background-subtracted light curves and errors were calculated as $(S-B / 5) / \Delta t$ and $\sqrt{(S+B / 25)} / \Delta t$, respectively.

\section{Binary Light Curve}

Figure 1 shows the Chandra light curves of EX Hya folded on the binary phase. A narrow partial eclipse is visible around $\phi_{98}=1.0$, as is the broad modulation centered on $\phi_{98} \sim 0.8$. We discuss the eclipse and the broad modulation separately in the following two sections.

\subsection{Eclipse}

We measured the position and duration of the eclipse by fitting $£(\lambda)$ for $\phi_{98} \in[0.9,1.1]$, using $10 \mathrm{~s}$ bins (or 0.0017 in $\phi_{98}$ ), with a second-order polynomial to represent the overall shape of the light curve, and a Gaussian to represent the eclipse (note that the bins used for the fit are much smaller than those shown in Fig. 1). We found for $£(1-20 \AA)$ that the eclipse is centered on $\phi_{98}=0.9947 \pm 0.0009$, which is slightly $(31 \mathrm{~s})$ before the time of eclipse predicted by the ephemeris of Hellier \& Sproats (1992), but well within its errors $\left(\sigma_{\phi_{98}}=\right.$ \pm 0.012 at the time of the observation). The eclipse has a FWHM of $(0.024 \pm 0.002) \times \phi_{98}$ or $141 \pm 12 \mathrm{~s}$, and has an eclipse deficit ${ }^{4}$ of $12.8 \% \pm 0.4 \%$. We find no evidence, at the $2 \sigma$ level, for any dependence of the time of eclipse, its width, or its deficit with wavelength (see Table 1). The FWHM of the eclipse is in good agreement with the measurement by Mukai et al. (1998) of $157 \pm 4 \mathrm{~s}$ based on 49 eclipses observed with $R X T E$. Furthermore, the eclipse deficit obtained from the Chandra data is in rough agreement with that obtained from EXOSAT, $20 \pm 4 \%$ (for the 1.5-3 keV band; Rosen et al. 1988) and Ginga, $18.2 \pm 1.7 \%$ (for the 1.7-2.8 keV band; Rosen et al. 1991).

\subsection{Bulge dip}

The broad modulation in the EX Hya binary light curve is thought to be due to absorption by the hot spot or bulge on the accretion disk. In our first attempt to describe the broad

\footnotetext{
${ }^{4}$ The eclipse deficit is defined by Rosen et al. (1988) as the total count rate during the eclipse, defined as 2 times the FWHM, compared to the count rate in two adjoining regions, each one FWHM wide, on either side of the eclipse.
} 
modulation, we modeled the light curves assuming a constant count rate and two Gaussians: a narrow one centered on $\phi_{98} \sim 1$ representing the eclipse discussed in the previous section, and a broad one centered on $\phi_{98} \sim 0.8$ representing the broad modulation. Table 1 lists the centroids, FWHM, and depth of the Gaussian fit to the broad modulation and the fits are shown in Figure 1.

As has been reported before (e.g., Rosen et al. 1991; Allan et al. 1998), the depth of the broad modulation becomes more pronounced for the longer wavelengths. However, we also find that the phase of the minimum of the broad modulation changes with wavelength. Both of these trends continue into the EUV wavelength band, as can be seen from Figures 2 and 3, which show the Chandra HETG $£(1-5 \AA), £(5-10 \AA), £(10-15 \AA)$, and $£(15-20 \AA)$ light curves and the EUVE DS $£(70-180 \AA)$ light curve. We argue below that the shift in the phase of the minimum of the broad modulation (hereafter $\phi_{98, \text { min }}$ ) with wavelength from $\mathrm{X}$-rays to the EUV is most likely due to a variation with $\phi_{98}$ in the photoelectric absorption characteristics of the absorbing material.

\subsection{Modeling the Bulge Dip}

In the following, we assume that the bulge dip can be modeled by a single column density that fully covers the source of X-ray and EUV light. In this case, the observed count rate $C\left(\lambda, \phi_{98}\right)$ can be written as:

$$
C\left(\lambda, \phi_{98}\right)=\bar{F}_{0}(\lambda) \exp \left[-N_{\mathrm{H}}\left(\phi_{98}\right) \sum_{i=1}^{28} A_{i} \sum_{j=1}^{i} \sigma_{i j}(\lambda) I_{i j}\left(\phi_{98}\right)\right]
$$

where $\bar{F}_{0}(\lambda)=F_{0}(\lambda) A R F(\lambda), F_{0}(\lambda)$ is the flux (in photons $\mathrm{cm}^{-2} \mathrm{~s}^{-1} \AA^{-1}$ ) of the source before it passes through the absorber, $A R F(\lambda)$ is the effective area of the instrument (in $\left.\mathrm{cm}^{2}\right), N_{\mathrm{H}}\left(\phi_{98}\right)$ is the $\mathrm{H}$ column density (in $\left.\mathrm{cm}^{-2}\right), A_{i}$ is the fractional abundance of element $i$ relative to $\mathrm{H}$, and $I_{i j}\left(\phi_{98}\right)$ and $\sigma_{i j}(\lambda)$ are respectively the ionization fraction and photoelectric cross section of ion $j$ of element $i$. We assume that the source spectrum is constant with $\phi_{98}$, that the abundances are solar (Grevesse \& Anders 1998), and that the photoelectric cross sections are as given by Verner \& Yakovlev (1995). With these assumptions, variations in $C\left(\lambda, \phi_{98}\right)$ with $\phi_{98}$ can be due to changes in the column density and/or the ionization state of the absorbing material.

To investigate the hypothesis that a changing ionization state could produce the EX Hya light curves, we consider two physical processes that affect the ionization state of the absorbing material: collisional ionization (CI) and photoionization (PI). For both processes we assume that the absorber is optically thin and has a homogeneous ionization balance (but 
see $\S 3.4)$. With these assumptions, the ionization balance is given for CI by the temperature $T$ and for PI by the ionization parameter $\xi=L / n R^{2}$, where $L$ is the luminosity (in $\operatorname{erg~s}^{-1}$ ), $n$ is the $\mathrm{H}$ density (in $\mathrm{cm}^{-3}$ ), and $R$ is the separation between the absorber and the source of the ionizing radiation (in $\mathrm{cm}$ ). In the CI case, we assume that the ionization fractions are as given by Mazzotta et el. (1998) as a function of $T\left(\phi_{98}\right)$. In the PI case, we calculated the ionization fractions with the CLOUDY photoionization code (Ferland 1996) as a function of $\xi\left(\phi_{98}\right)$.

In addition to $L, n$, and $R$, it is necessary in CLOUDY to specify the shape of the ionizing spectrum. For this, we used a combination of (1) a multi-temperature APEC (Smith et al. 2001) thermal plasma model fit to the Chandra data, representing the emission from the accretion columns, (2) a blackbody spectrum to represent the white dwarf surface (radius $R=10^{9} \mathrm{~cm}$ and temperature $T=10000 \mathrm{~K}$, Eisenbart et al. 2002), and (3) a blackbody spectrum to represent the portion of the white dwarf surface that is heated by emission from the accretion columns (effective radius $R=6 \times 10^{8} \mathrm{~cm}$ and temperature $T=25000 \mathrm{~K}$, Eisenbart et al. 2002). We thus assume that the absorber is photoionized from only one side by the white dwarf and accretion columns. The ionizing radiation from the disk and the companion are negligible and can be ignored.

To determine the spectral shape in the Chandra wavelength band, we fitted the APEC model to the part of the observation least affected by absorption, i.e., $\phi_{67} \in[0.9,1.3]$ and $\phi_{98} \in[1.0,1.6]$. The APEC model consists of 35 temperature components ranging from $1.2 \times 10^{5} \mathrm{~K}$ to $2.3 \times 10^{8} \mathrm{~K}$ with an emission measure $\left(\mathrm{EM}=\int n_{\mathrm{e}} n_{\mathrm{H}} \mathrm{d} V\right)$ distribution $E M(T) \propto$ $T^{1 / 2}$ for $T>10^{7} \mathrm{~K}$ and $E M(T) \propto T^{3 / 2}$ for $T<10^{7} \mathrm{~K}$, i.e., the typical emission measure distribution for a plasma cooling mainly through thermal bremsstrahlung for $T>10^{7} \mathrm{~K}$ and mainly through line emission below $10^{7} \mathrm{~K}$. To make sure that the model correctly reproduces the ultraviolet and far ultraviolet flux observed in EX Hya, we added an additional temperature component at $T=3.1 \times 10^{5} \mathrm{~K}$ with an emission measure of $2 \times 10^{52} \mathrm{~cm}^{-3}$ to produce the O VI lines measured by ORFEUS II (Mauche 1999).

Consistent with Eisenbart et al. (2002), we set the total luminosity $L=10^{32} \mathrm{erg} \mathrm{s}^{-1}$ between 1 Ryd and 1000 Ryd, which results in luminosities of $13,3,1.5$, and $1.9 \times 10^{-11} \mathrm{erg} \mathrm{cm}^{-2}$ $\mathrm{s}^{-1}$ in the $\geq 1.0 \mathrm{keV}, 0.28-1.0 \mathrm{keV}, 0.067-0.028 \mathrm{keV}$, and $0.0136-0.067 \mathrm{keV}$ bands, respectively. The ionization parameter $\log \xi$ was sampled from -4 to 4 in steps of $\Delta \log \xi=0.25$, $R$ was fixed at $10^{10} \mathrm{~cm}$ (see $\S 3.4$ ), and the density $n$ varied accordingly. The choices of $R$ and $n$ are arbitrary since it is only $\xi$ that detemines the ionization fraction. Since we are interested only in the optically thin case, we adopted the ionization balance of the first 'zone/shell' in the CLOUDY calculations, i.e, the inner edge of the absorber. 


\subsection{Fit Results}

To make the fitting process simpler we (1) normalize the light curves to one for $\phi_{98} \in$ $[0.2,0.4]$, where we find no appreciable absorption, (2) use bins $0.02 \times \phi_{98}$ in size, (3) increase the errors on the EUVE light curve by a factor of four, to make them similar to those of the Chandra light curves (so that they do not dominate the fitting process), and (4) removed the white dwarf eclipse from the light curves, i.e., we do not fit between $\phi_{98} \in[0.98,1.04]$.

Figure 2 shows the result of fitting the amount of absorption (Eq. 1) to the Chandra and $E U V E$ light curves simultaneously, for each $\phi_{98}$ bin, using as free parameters the column density $N_{\mathrm{H}}$ and the temperature $T$ for the CI case and the ionization parameter $\xi$ for the PI case. The figure clearly shows that, for the region where the absorption is appreciable, i.e., $\phi_{98} \in[0.6,1.0],(1)$ the variation of the column density can be described roughly by a Gaussian in $\phi_{98}$ and (2) $T$ and $\xi$ decline roughly linearly with $\phi_{98}$ (possibly hitting a floor around $\phi_{98}=0.85$, after which they remain fairly constant). The fits are poorly determined outside the $\phi_{98} \in[0.6,1.0]$ interval due to the fact that no appreciable absorption is present, so we do not show the fit parameters in this region.

Based on the trends shown in Figure 2 we fitted $T$ and $\xi$ by a linear function:

$$
\log X\left(\phi_{98}\right)=c_{0}+c_{1} \phi_{98}
$$

where $X$ represents either $T$ or $\xi$, and fitted $N_{\mathrm{H}}$ by a Gaussian function:

$$
N_{\mathrm{H}}\left(\phi_{98}\right)=N_{\mathrm{H}, 0} \exp \left[-\left(\phi_{98}-\phi_{N_{\mathrm{H}}}\right)^{2} / 2 \sigma_{N_{\mathrm{H}}}^{2}\right]
$$

Table 2 summarizes the results of the fits, which are shown in Figure 3. While these simple models do not fit the light curves perfectly, they reproduce, with a minimum number of degrees of freedom, the essential features of the light curves, including the change of $\phi_{98, \min }$ with wavelength.

Note that we find a maximum column density $N_{\mathrm{H}, 0} \sim 4 \times 10^{21} \mathrm{~cm}^{-2}$, which is a factor four higher than most values obtained in previous studies (e.g., Hurwitz et al. 1997). This difference is readily explained by the fact that the other studies assumed neutral absorbers, while our model of the Chandra and EUVE light curves requires a partially ionized absorber. Specifically, we require an absorber with no H I or He I opacity; in our model, most of the absorption in the EUVE wavelength band is due to He II.

Given the parameters inferred for our fits to $N_{\mathrm{H}}\left(\phi_{98}\right)$, it is possible to draw a schematic picture of the location and extent of the absorbing material in EX Hya. In the sketch shown in Figure 4, we have assumed that the inner edge of the disk lies at $R_{\text {in }} \sim 0.7 \times 10^{10} \mathrm{~cm}$ (Allan et al. 1998) and that the outer edge of the disk lies at $R_{\text {out }} \sim 1.5 \times 10^{10} \mathrm{~cm}(75 \%$ of the white 
dwarf Roche lobe radius of $2 \times 10^{10} \mathrm{~cm}$, Beuermann et al. 2003; Hoogerwerf et al. 2004). The absorber can be drawn on this figure if we assume that its density $n=N_{\mathrm{H}}\left(\phi_{98}\right) / D\left(\phi_{98}\right)$ is constant with $\phi_{98}$ and its thickness $D\left(\phi_{98}\right)=R_{2}-R_{1}$, where $R_{1} \geq R_{\text {in }}$ and $R_{2} \leq R_{\text {disk }}$. Two representative absorbers are shown in the figure for $R_{1}=R_{\text {in }}$ and $n=5 \times 10^{11} \mathrm{~cm}^{-3}$ and $R_{1}=0.75 R_{\text {disk }}$ and $n=1 \times 10^{12} \mathrm{~cm}^{-3}$.

In the PI case, additional constraints are imposed on the absorber by the definition of the ionization parameter. Eliminating $n$ between the relationships $\xi=L / n R^{2} \sim 3$ and $N_{\mathrm{H}}=n D \sim 3 \times 10^{21} \mathrm{~cm}^{-2}$ gives $D_{10} \sim 0.9 R_{10}^{2}$, where $D$ and $R$ are expressed in units of $10^{10} \mathrm{~cm}$. This is further constrained by the requirement $D / R \ll 1$ so that the ionization parameter does not vary significantly from the front to the back of the absorber. These constraints are satisfied only if the absorber is very near the white dwarf: for $R \leq 0.3 R_{\text {in }}$, $D / R \lesssim 0.3$ and $n \gtrsim 4 \times 10^{12} \mathrm{~cm}^{-3}$. However, this analysis requires that the PI absorber be optically thin to the ionizing radiation, while Figure 3 shows that during the dip the EUVE light curve is extinguished by approximately 95\%, corresponding to an optical depth $\tau \approx 3$.

\subsection{Optically Thick PI Model}

To allow the possibility of an optically thick PI absorber, we ran a set of full CLOUDY models to account for the dilution of the radiation field and the resulting change of the ionization balance with depth into the absorber. The dashed curve in the middle panel of Figure 3 shows the full CLOUDY light curves based on our best fit paramaters of the optically thin model for $R=10^{10} \mathrm{~cm}$. This clearly shows that including the optical depth effects and physical size of the absorber results in more absorption, particularly at the longer wavelengths. We are able to produce full CLOUDY models that fit our light curves as well as we did for the CI and optically thin PI models, but these models require that the inner radius of the absorber be at $R \sim 0.4 \times 10^{10} \mathrm{~cm}$, i.e., smaller than the inner radius of the accretion disk. While the accretion curtain is a source of absorption at this close distance to the white dwarf, this absorption should be visible only on the white dwarf period and not on the binary period. We thus conclude that the white dwarf in EX Hya is not luminous enough to ionize the absorber by photoionization processes alone.

\subsection{Heating of the Absorber}

We can solve the problems with the PI model described above by imposing a base temperature to the absorber of order $10^{5} \mathrm{~K}$. This ensures that no H I and He I and almost 
no He II is present in the absorber, as is required by the ionization balances found by the CI and PI models. This is crucial, since even small traces of these ions result in large amounts of absorption in the EUVE waveband. Since this base temperature is significantly higher than the temperature of the outer disk (3000-7000 K, Frank et al. 1981; Eisenbart et al. 2002), we conclude that viscous heating alone is insufficient to keep the absorber hot. Other sources of heat, such as shocks due to the interaction between the accretion stream and the accretion disk, are needed.

\section{Discussion}

As shown above, the dominant source of ionizing radiation in EX Hya, the white dwarf and accretion columns, are not sufficiently luminous to ionize the accretion disk bulge by photoionization alone. Therefore, the bulge must be ionized through a different process and, assuming that it is in, or close to, CI equilibrium, its temperature is of order $\log T(\mathrm{~K}) \sim 5.3$

or $T \sim 200,000 \mathrm{~K}$. Furthermore, to reproduce the shift of $\phi_{98, \min }$ with wavelength, the bulge must have a temperature gradient that steadily decreases from $\log T(\mathrm{~K}) \sim 5.5$ at $\phi_{98} \sim 0.6$ to $\log T(\mathrm{~K}) \sim 5.2$ at $\phi_{98} \sim 0.9$. We speculate that this temperature gradient is caused by the interaction, e.g., shocks, between the stream of accreting material from the companion and the accretion disk.

Our simple analysis in $\S 3.4$ places the location of this interaction region on the disk at $\phi_{98} \approx 0.6-1.0$ corresponding to a range of angles of approximately $0^{\circ}-130^{\circ}$ from the line joining the two stars. The location and shape of the absorber is uncertain because we measure only the column density along the line of sight, although Figure 4 shows two possibilities that are consistent with our data. In interpreting this figure, it must be kept in mind that what we are seeing in the X-ray and EUV wavelength bands is only that portion of the accretion bulge that rises an angle of $13^{\circ}$ above the orbital plane.

Hydrodynamical simulations of close binary systems show that the inflowing material from the companion star forms a spiral-like structure in the accretion disk instead of the canonical hot spot on the edge of the disk (e.g., Bisikalo et al. 1998; Makita, Miyawaki, \& Matsuda 2000; Sato, Sawada, \& Ohnishi 2003). The location of such spiral structures appears to coincide with the position of the EX Hya absorber. Furthermore, simulations show that shocks occur in regions where the inflowing material interacts with the accretion disk. These shocks might be responsible for the heating needed in our EX Hya absorber model. Unfortunately, most of the hydrodynamic simulations do not include the effects of heating and cooling on the accretion disk structure (but see, Sato et al. 2003). The EX Hya results presented in this paper provide an excellent constraint for new hydrodynamic simulations of 
close binary systems.

\section{Visibility of Absorber}

A hot absorber like the one in EX Hya will generate its own emission, mainly in the UV band, the strength of which is given by the emission measure. Unfortunately, we know only the column density and temperature along a slice through the absorber. It is unknown what the total volume of the absorber is and whether the temperature distribution is similar throughout the absorber. Furthermore, the emission measure depends on the density of the absorber, which is also an unknown. Estimates and assumptions for all these quantities can be made, but the result will be highly unreliable. To our knowledge there is no mention in the literature that the absorber has ever been observed in emission.

We do however, see evidence of the absorber in ORFEUS II data (Mauche 1999) and, more clearly, in FUSE data, for which the analysis is in progress, as line absorption features of the O VI $\lambda \lambda 1032,1038$ doublet lines and the C III $\lambda 977, \lambda 1175$ lines at binary phases $0.6<\phi_{98}<1.0$. The absorption features are not visible at other binary phases. This range of binary phases agrees well with the absorber described in this paper. Furthermore, the fact the O VI shows line absorption confirms that the absorber contains highly ionized oxygen.

\section{Short Wavelength Absorption}

Figure 3 shows that both the CI and PI model fits do not perform very well in the $1-5 \AA$ band and the 5-10 $\AA$ band: both models tend to underpredict the amount of absorption. We found that adding a second absorber to our model improves the fit: it lowers the reduced $\chi^{2}$ from $\sim 4$ to $\sim 2$ (see bottom panel Fig. 3). The second absorber was modeled with a Gaussian for the column density and a single temperature or ionization parameter. The results are that this absorber needs to be highly ionized $[\log T(\mathrm{~K}) \sim 7$ or $\log \xi \sim 2.7]$ and have a large column $\log N_{\mathrm{H}}\left(\mathrm{cm}^{-3}\right) \sim 23$. Using similar arguments as in $\S 3.4$ we can constrain the position of the absorber, in the PI case, to be very close $\left(R<4 \times 10^{8} \mathrm{~cm}\right)$ to the source of the ionizing emission, i.e., basically at the shock front. It is hard to understand why this feature would appear on the binary period rather than the white dwarf period. If real, the second absorber is most likely to be near the inner edge of the accretion disk, somehow

uncoupled to the magnetic field of the white dwarf. A long observation of EX Hya could confirm the existence of a second absorber and perhaps better constrain its location. 


\section{Conclusions}

We report a new feature in the binary light curves of the magnetic cataclysmic variable EX Hya. Based on Chandra HETG and EUVE DS data we have shown that the phase of the minimum in the broad modulation, associated with the bulge or hot spot on the accretion disk, increases with wavelength. Collisional ionization and photoionization models explain this characteristic as a change in ionization state of the bulge plasma with binary phase. However, the ionizing radiation originating on the white dwarf and its accretion columns is insufficient to account for the ionization state of the bulge. Moreover, the required ionization state also excludes viscous heating in the accretion disk as the main source of ionization. We thus conclude that the bulge plasma is heated by shocks resulting from the interaction between the inflowing material from the companion and the accretion disk.

We thank John Raymond for comments and suggestions and Yair Krongold and Jonathan Slavin for help setting up with CLOUDY. We also thank Gary Ferland for a bug fix in and advice on CLOUDY related to this paper and the referee for a critical reading of the manuscript. We thank H. Tananbaum for the generous grant of Director's Discretionary Time that made possible the Chandra observations of EX Hya. This research has made use of data obtained from the High Energy Astrophysics Science Archive Research Center

(HEASARC), provided by NASA's Goddard Space Flight Center. We acknowledge support from NASA through Chandra grants GO2-3018X and GO3-4017X. NB was supported by NASA contract NAS8-39073 to the Chandra X-ray Center. CWM's contribution to this work was performed under the auspices of the U.S. Department of Energy by University of California Lawrence Livermore National Laboratory under contract No. W-7405-Eng-48.

\section{REFERENCES}

Aizu, K. 1973, Prog. Theor. Phys., 49, 1184

Allan, A., Hellier, C. H., \& Beardmore, A. 1998, MNRAS, 295, 167

Belle, K. E., Howell, S. B., Sirk, M. M., \& Huber, M. E. 2002, ApJ, 577, 359

Belle, K. E., Howell, S. B., Sion, E. M., Long, K. S., \& Szkody, P. 2003, ApJ, 587, 373

Beuermann, K., Harrison, The. E., McArthur, B. E., Benedict, G. F., \& Gänsicke, B. T. 2003, A\&A, 412, 821 
Bisikalo, D. V., Boyarchuk, A. A., Chechetkin, V. M., Kuznetsov, O. A., \& Molteni, D. 1998, MNRAS, 300, 39

Bowyer S., \& Malina R. F., 1991, in Extreme Ultraviolet Astronomy, ed. Malina R. F., Bowyer S., Pergamon, New York, p. 397

Bowyer S., Lieu R., Lampton M., Lewis J., Wu X., Drake J. J., \& Malina R. F., 1994, ApJS, 93,569

Eisenbart, S., Beuermann, K., Reinsch, K., \& Gänsicke, B. T. 2002, A\&A, 382, 984

Ferland, G. 1996, Hazy, a Brief Introduction to Cloudy, University of Kentucky Department of Physics and Astronomy Internal Report

Frank, J., King, A. R., Sherrington, M. R., Giles, A. B., \& Jameson, R. F. 1981, MNRAS 196, 921

Fujimoto, R., \& Ishida, M. 1997, ApJ, 474, 774

Grevesse, N., \& Anders, E. 1998, Space Sci. Rev., 85, 161

Hellier, C., \& Sproats, L. N. 1992, IBVS, 3724, 1

Hoogerwerf, R., Brickhouse, N. S., \& Mauche, C. W. 2004, ApJ, 610, 411

Hurwitz, M., Sirk, M., Bowyer, S., \& Yuan-Kuen, K. 1997, ApJ, 477, 390

Kylafis, N. D., \& Lamb, D., Q. 1982, ApJS, 48, 239

Mauche, C. W. 1999, ApJ, 520, 822

Mauche, C. W. 2002, in ASP Conf. Ser., 264: Continuing the Challenge of EUV Astronomy: Current Analysis and Prospects for the Future, ed. S. B. Howell, J. Dupuis, D. Golombek, F. M. Walter, and J. Cullison, 75

Makita, M., Miyawaki, K., \& Matsuda, T. 2000, MNRAS, 316, 906

Mazzotta, P., Mazzitelli, G., Colafrancesco, S., \& Vittorio N. 1998, A\&AS, 133, 403

Mukai, K. 1999, in ASP Conf. Ser., 157, Annapolis Workshop on Magnetic Cataclysmic Variables, ed. C. Hellier, \& K. Mukai (San Fransisco: ASP), 33

Mukai, K., Ishida, M., Osborne, J., Rosen, S., \& Stavroyiannopoulos, D. 1998, in ASP Conf. Ser., 137, Wild Stars in the Old West, ed. S. Howell, E. Kuulkers, \& C. Woodward (San Fransisco: ASP), 554 
Rosen, S. R., Mason, K. O., \& Córdova, F. A. 1988, MNRAS, 231, 549

Rosen, S. R., Mason, K. O., Mukai K., \& Willams, O. R. 1991, MNRAS, 249, 417

Sato, J., Sawada, K., \& Ohnishi, N. 2003, MNRAS, 342, 593

Smith, R. K., Brickhouse, N. S., Liedahl, D. A., \& Raymond, J. C. 2001, ApJ, 556, L91

Sterken, C., Vogt, N., Freeth, R., Kennedy, H. D., Marino, B. F., Page, A. A., \& Walker, W. S. G. 1983, A\&A, 118, 325

Verner, D. A., \& Yakovlev, D. G. 1995, A\&AS, 109, 125

Vogt, N., Krzeminski, W., \& Sterken, C. 1980, A\&A, 85, 106

Wood, J., Horne, K., Berriman, G., Wade, R., O’Donoghue, D., \& Warner, B. 1986, MNRAS, 219,629 


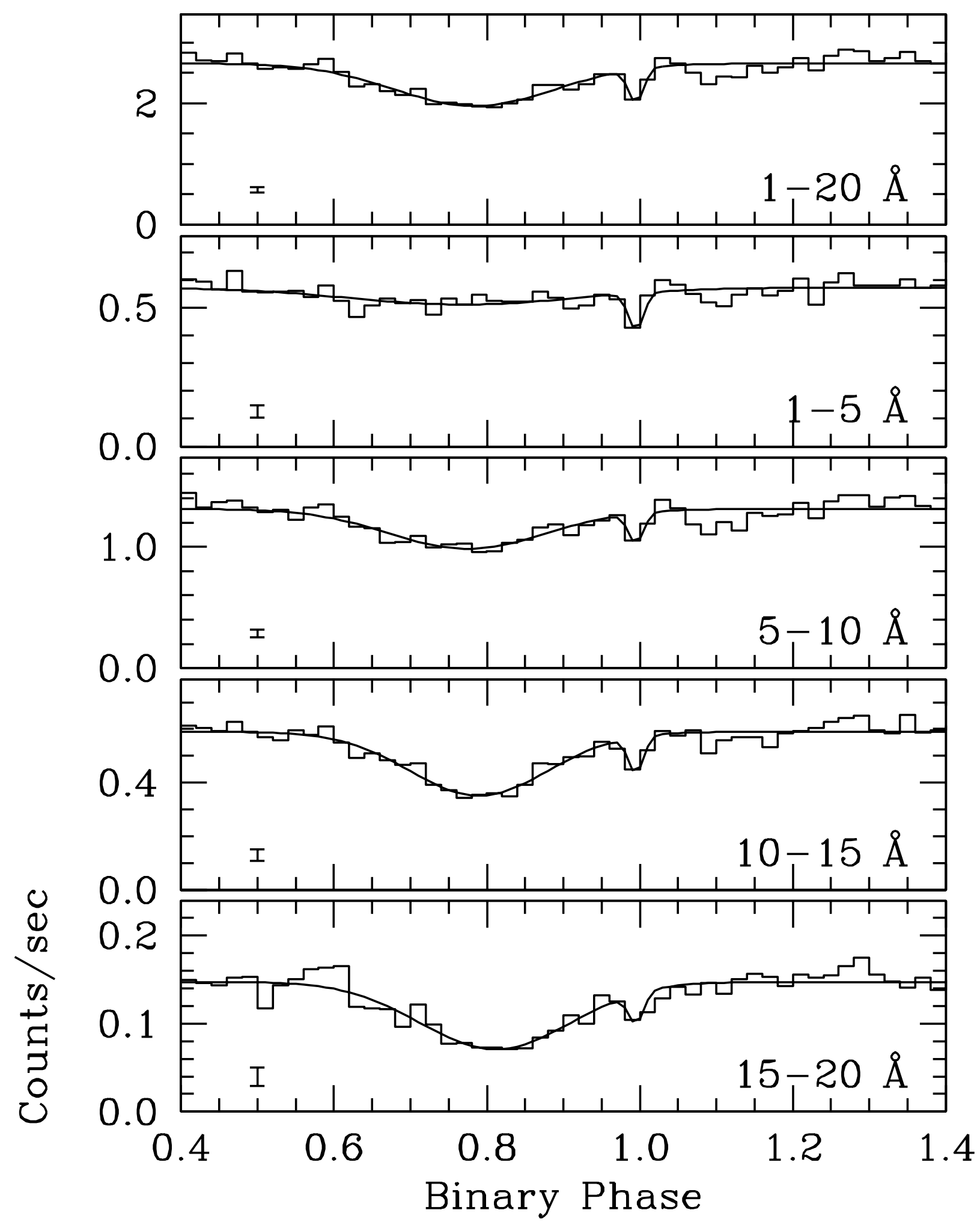

Fig. 1.- EX Hya binary light curves in the five Chandra first-order HETG wavelength bands. The average error of each light curve is indicated in the lower left corner of each panel. Each panel also shows, as a solid line, the model fit to the data discussed in $\S 3.2$, with the fit parameters in Table 1. 

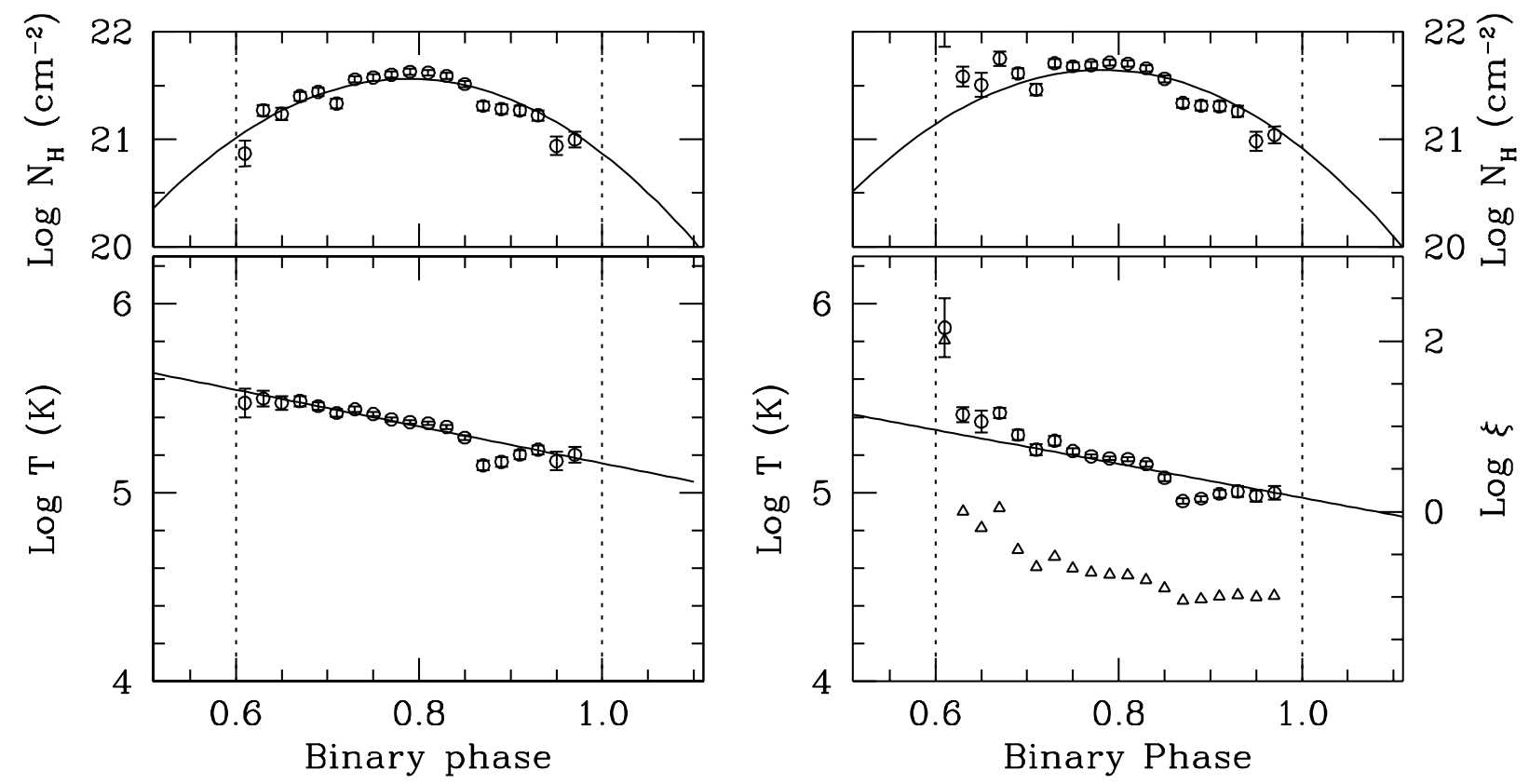

Fig. 2.- Left (right) panels The best fitting $N_{\mathrm{H}}$ and $T(\xi)$ for the collisional (photo) ionization model. The vertical dotted lines in the lower panels indicate the region for which the absorption is appreciable and can be fitted reliably. The open circles denote the temperature (ionization parameter) for the fit. The open triangles denoted the temperature of the gas due to heating by the PI process. The solid curves show the best fit temperature (ionization parameter) model (see $\S 3.4)$. 


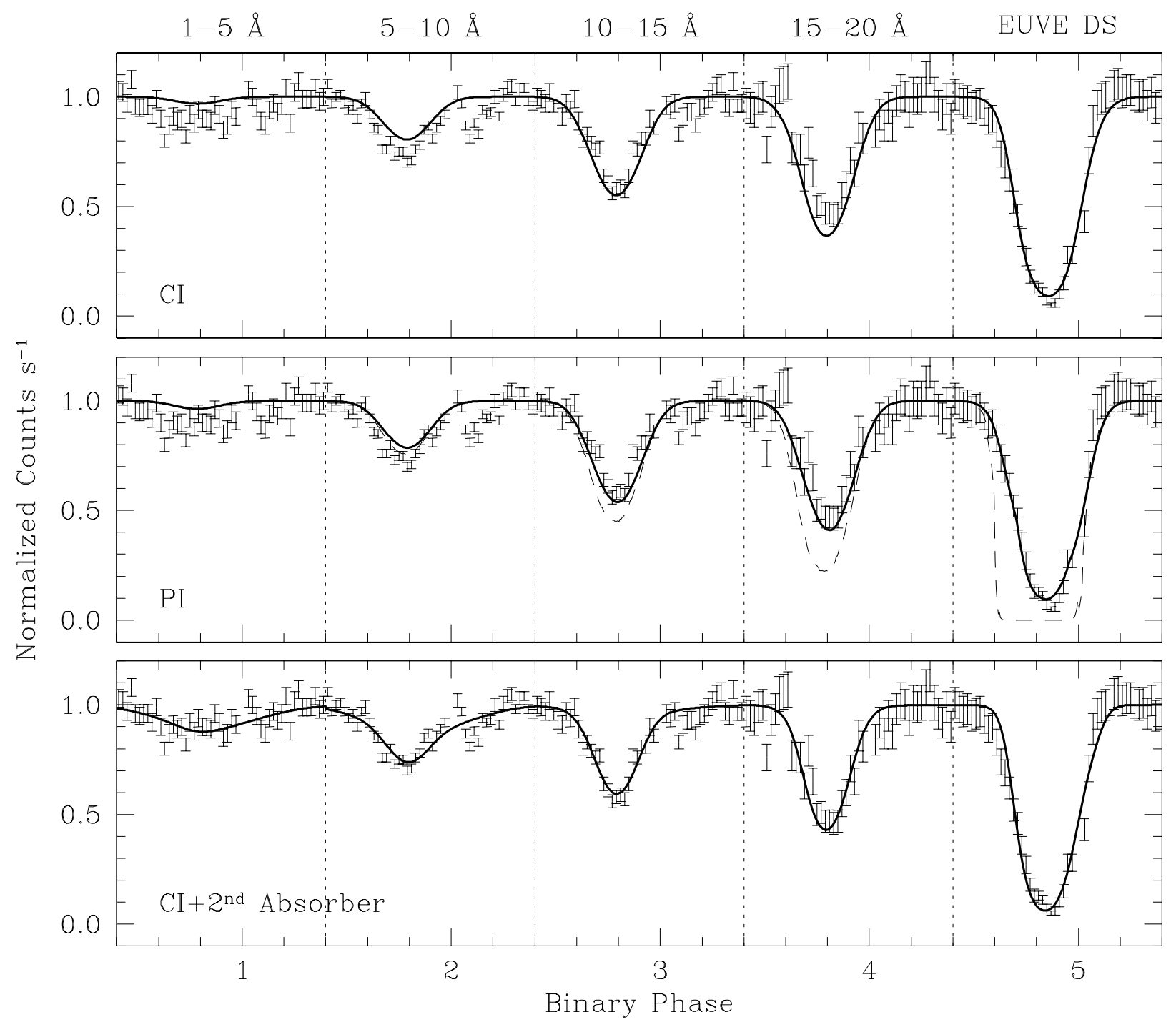

Fig. 3.- EX Hya binary light curves in the four Chandra HETG and EUVE DS wavelength bands. The dotted vertical lines separate the different wavelength bands. Top panel shows the best fitting CI model and middle panel shows the best fitting PI model. The dashed line in the middle panel shows the light curves predicted by the full CLOUDY calculation discussed in $\S 3.5$. Bottom panel shows the light curve produced by the CI model with an additional absorber. 


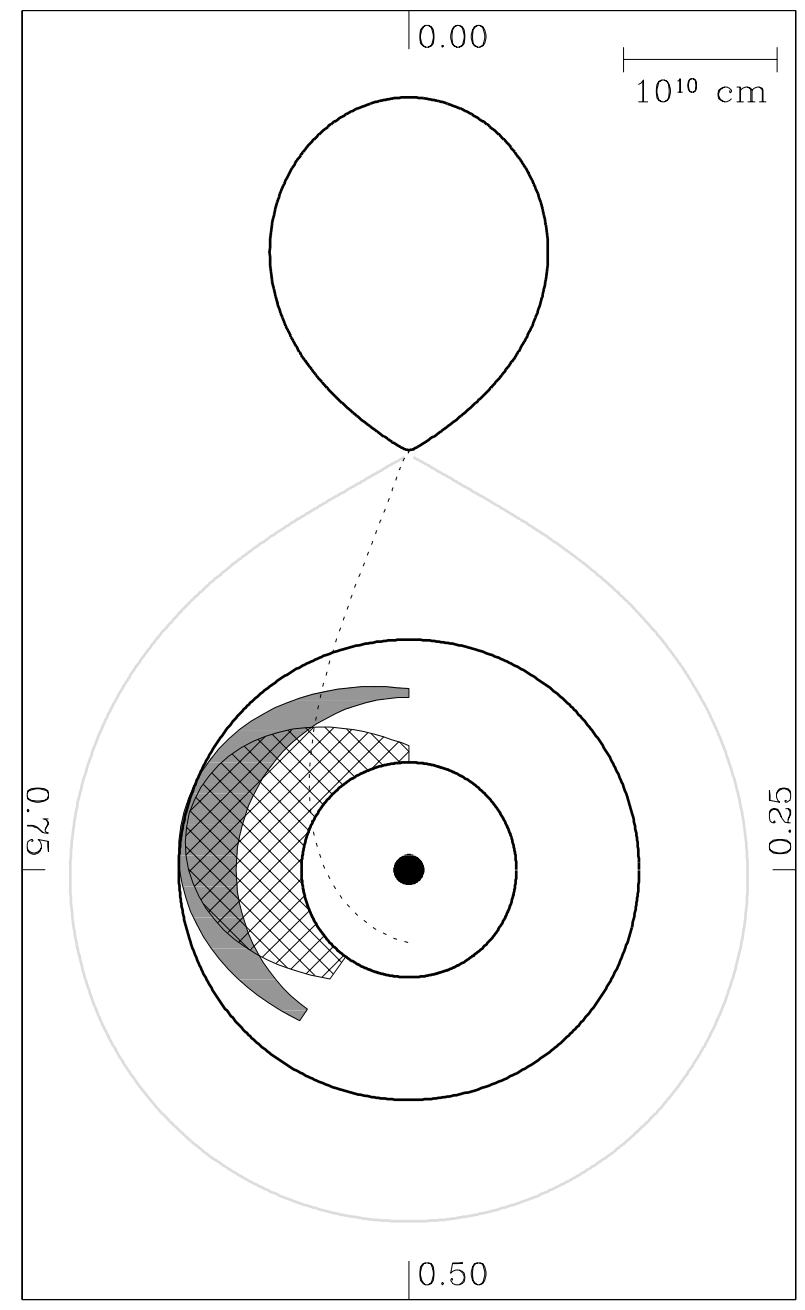

Fig. 4. - Schematic of the EX Hya binary, showing the companion, the white dwarf (small solid circle), the Roche limit of the white dwarf (light gray curve), the inner and outer disk radii (circles), the ballistic stream (dotted curve), and the location of the absorber for the two cases discussed in $\S 3.4$. The binary phases are indicated, as is the scale. 
Table 1. Binary Light Curve Fit Parameters

\begin{tabular}{|c|c|c|c|c|c|c|}
\hline \multirow[b]{2}{*}{$\begin{array}{l}\text { Wavelength Range } \\
(\AA)\end{array}$} & \multicolumn{3}{|c|}{ Broad Modulation } & \multicolumn{3}{|c|}{ Eclipse } \\
\hline & $\begin{array}{c}\text { Centroid } \\
\left(\phi_{98}\right)\end{array}$ & $\begin{array}{c}\text { FWHM } \\
\left(\phi_{98}\right)\end{array}$ & $\begin{array}{l}\text { Depth } \\
(\%)\end{array}$ & $\begin{array}{c}\text { Centroid } \\
\left(\phi_{98}\right)\end{array}$ & $\begin{array}{c}\text { FWHM } \\
\left(\phi_{98}\right)\end{array}$ & $\begin{array}{l}\text { Deficit } \\
(\%)\end{array}$ \\
\hline $1-20$ & $0.784 \pm 0.002$ & $0.250 \pm 0.006$ & $26.3 \pm 0.5$ & $0.995 \pm 0.001$ & $0.024 \pm 0.002$ & $12.8 \pm 0.4$ \\
\hline $1-5$ & $0.769 \pm 0.015$ & $0.354 \pm 0.050$ & $10.7 \pm 1.0$ & $0.994 \pm 0.001$ & $0.020 \pm 0.004$ & $17.5 \pm 1.2$ \\
\hline $5-10$ & $0.775 \pm 0.003$ & $0.244 \pm 0.009$ & $25.0 \pm 0.7$ & $0.996 \pm 0.001$ & $0.024 \pm 0.003$ & $11.9 \pm 0.6$ \\
\hline $10-15$ & $0.790 \pm 0.003$ & $0.214 \pm 0.007$ & $40.3 \pm 1.0$ & $0.992 \pm 0.002$ & $0.025 \pm 0.005$ & $12.2 \pm 0.9$ \\
\hline $15-20$ & $0.811 \pm 0.004$ & $0.232 \pm 0.012$ & $52.0 \pm 1.9$ & $1.000 \pm 0.002$ & $0.017 \pm 0.008$ & $14.4 \pm 2.6$ \\
\hline
\end{tabular}

Table 2. Collisional and Photoionization Model Parameters

\begin{tabular}{lcccc}
\hline \hline & & & \multicolumn{2}{c}{ Including } \\
& CI & PI & CI & PI \\
\hline $\log N_{\mathrm{H}, 0}$ & $21.59(1)$ & $21.67(1)$ & $21.50(1)$ & $21.55(2)$ \\
$\phi_{N_{\mathrm{H}}}$ & $0.786(3)$ & $0.782(3)$ & $0.790(3)$ & $0.764(4)$ \\
$\sigma_{N_{\mathrm{H}}}$ & $0.108(3)$ & $0.108(2)$ & $0.094(2)$ & $0.097(2)$ \\
$c_{0}$ & $6.13(2)$ & $2.15(3)$ & $6.27(1)$ & $3.34(3)$ \\
$c_{1}$ & $0.97(4)$ & $1.99(4)$ & $1.23(2)$ & $3.66(5)$ \\
$\log \hat{N}_{\mathrm{H}, 0}$ & & & $22.79(8)$ & $23.1(1)$ \\
$\phi_{\hat{N}_{\mathrm{H}}}$ & & & $0.85(1)$ & $0.87(1)$ \\
$\sigma_{\hat{N}_{\mathrm{H}}}$ & & & $0.23(2)$ & $0.22(2)$ \\
$c_{3}$ & & & $7.04(4)$ & $2.69(7)$ \\
$\chi^{2} / \nu$ & 4.15 & 3.79 & 2.32 & 2.09 \\
\hline
\end{tabular}

